\title{
GENITAL TUBERCULOSIS IN MALE CHILDREN *
}

\section{J. DELLINGER BARNEY, M.D.}

\section{BOSTON}

Although tuberculosis in one form or another is common in children, this disease seems to attack the genital and urinary organs comparatively rarely, a point on which most investigators are in agreement. There is reason to believe, however, that tuberculosis of the genital tract is not so uncommon as has been supposed, the difficulty lying in the fact that it may be mild, that a spontaneous cure may be effected, and that because of these facts the condition may either be overlooked or ascribed to an infection by organisms other than the tubercle bacillus. Be that as it may, large numbers of cases have not been reported.

Poissonnier, ${ }^{1}$ in a very complete review of the subject of infantile genital tuberculosis, collected ninety-one cases from various sources, the greatest number, forty-four, having been observed by Broca among 46,000 juvenile hospital inmates. Since this time various writers have reported from one to three or four cases each, so that it would not appear that advances in diagnostic acumen were reaping a much richer harvest.

It is not, therefore, surprising to find that the Massachusetts General Hospital has shared in this comparative paucity of material. A search of the records from 1872 to date reveals a total of eleven such cases, among 401 admissions (including reentries) for this condition, a percentage of 2.74 .

Various statements are made to the effect that the disease affects particularly very young children, decreasing in frequency as puberty approaches. Poissonnier, among eighty-nine cases, found forty-two occurring before the second year, forty-seven between the second and fifteenth year. Among our eleven cases the youngest child was 9 months old, the other children being, respectively, 18 months, 2 years, 21/2 years, 5 years, 6 years, 61/2 years, 7 years, 8 years, 12 years and 14 years of age. That the nursing infant is not spared is shown by two such patients reported by Swoboda, ${ }^{2}$ and we are even informed that a case of this disease in a fetus at term has been observed by Drechsfeld (quoted by Poissonnier ${ }^{1}$ ).

\footnotetext{
* Received for publication, July 9, 1921.

* From the Genito-Urinary and Children's Medical Departments of the Massachusetts General Hospital.

1. Poissonnier: Gaz. des hôp., 1907, pp. 375-380.

2. Kirmisson: Rev. gen. de clin. et de Therap. 32:641-644, 1918.
} 
In our series of eleven cases both epididymes were found to be involved at the time of entrance in one case (an infant aged 9 months); in another case (a boy aged 8 years) the second side became involved twenty-seven months after removal of the epididymis first affected, while in a third case the involvement of the second side took place within three months after excision of its tuberculous mate (orchidectomy). These facts seem to be in accord with the views of other writers, especially Kirmission ${ }^{2}$ and Poissonnier, ${ }^{1}$ the latter having collected only seven bilateral infections out of forty-two cases. Bilaterality of the disease in children is therefore far less common than in adults. My own statistics, published some years ago, ${ }^{3}$ showed that at the time of entrance to the hospital both sides were involved in 29.3 per cent. of 150 adult cases, while König found bilateral lesions in 75 per cent., and von Brun found both sides involved in 38 per cent. At the Tübingen clinic, 29 per cent. of 111 cases were found to be bilateral.

The remaining eight cases in our series showed about an equal distribution of the disease between the left and right sides.

Kirmission ${ }^{2}$ states that in adults the testicle itself is often intact, whereas in children this organ is frequently diseased. On the other hand, Vignard and Thévenot ${ }^{4}$ assert that the testicle and epididymis are attacked simultaneously, a view which is shared by Poissonnier, ${ }^{1}$ who states that the burden of infection is shared equally by these organs.

Our own experience shows that in only two instances was the testis itself definitely tuberculous, but it should be stated that the pathologic reports, more particularly those of an earlier day, are very vague as to the differentiation between testicle and epididymis. I believe that there is much less frequently an involvement of the infantile than of the adult testicle, and that in most cases it is no more necessary to remove the testicle of a child than that of an adult. Indeed, it may be said that the removal of this organ from patients under the age of puberty should be done only as a last resort, with much less impunity than in the adult, owing to the demand made on its invaluable internal secretion as puberty approaches.

While the formation of a scrotal abscess with spontaneous rupture and the establishment of a fistula may occur in children as in adults, it certainly is a far less common phenomenon. I have already showed that such an event takes place in over 76 per cent. of adult cases, whereas in the children under discussion scrotal fistula was found only three times.

3. Barney: Mod. Urol. 1:489-533.

4. Vignard and Thévenot: Ann. d. méd. et de chir. Enf. 15:561, 1911. 
While it is unfortunate that our records definitely state that the vas deferens was involved in only two instances, there is no reason to believe that the seminal duct of children is less frequently invaded by tuberculosis than that of the adult. In the latter, such an involvement is found in the great majority of cases, and according to Poissonnier ${ }^{1}$ the vas was definitely tuberculous in eighteen out of twenty-eight children in whom this point was noted.

It is unfortunate also that in so few cases is not only the condition of the vas deferens, but also that of the prostate and seminal vesicles recorded. Since so much hinges on the knowledge of the condition of the latter organs not only from the scientific, but also from the prognostic viewpoint, it is disappointing to find that no mention whatever is made of these organs by many observers. While it is true that in children rectal examination is not easy and may be productive of pain, the facts can readily be ascertained if and when the child is anesthetized for operation.

The records of our cases compare favorably with others in this respect, definite statements as to the condition of the prostate or seminal vesicles or both having been made in six cases, twice at necropsy and in the other four either at or after operation. Of the latter, in a boy aged 8 years, the seminal vesicles were recorded as "palpable" and "very tender" before operation, whereas over two years later, tuberculosis of the second epididymis and of one kidney having meantime intervened, the record states that the "prostate is normal and the vesicles are not felt." In a boy aged 14, with unilateral epididymitis of short duration it is recorded that "nothing is felt in the prostate," and in a child 5 years of age, with a tuberculous epididymitis of over two years' duration on the one side, and of from three to four months' standing on the other, we are told that the rectal examination was "negative." Finally, a boy aged 7 , having a unilateral orchidectomy in 1905, was examined at the hospital in 1911 and was found to have a normal prostate and seminal vesicles and the remaining testis and epididymis free from disease.

Although these cases will be dwelt on later at greater length I may say that in two cases seen at necropsy, one with bilateral disease and unoperated, showed an extensive involvement of the seminal vesicles and a slight and early invasion of the prostate, whereas the second patient having had an operation on one tuberculous testicle died, twenty-six days later, and was found to have a normal prostate and seminal vesicles. Thus five out of six patients are known to have had no demonstrable lesion of the prostate or seminal vesicles.

Kantorowicz, in fifty-seven cases of epididymal tuberculosis in children, found the prostate involved but twice. Broca, who examined all of his forty-four cases, is reported never to have found a lesion 
of the prostate or vesicle. In support of these views Kirmission states that it is much more rare than in the adult to find lesions of the prostate and seminal vesicles in children. On the other hand, Vignard and Thévenot state that in these young children "the vesicles and prostate are often the seat of indurated nodules or of suppuration." There seems, therefore, to be a difference of opinion but I am inclined to believe that the question of prostatic and vesicular tuberculosis could be settled definitely if careful routine examinations were made of these organs. It is worthy of note, however, that in a careful review of the literature of this subject no mention whatever is made of any instances of tuberculosis of the prostate or seminal vesicles being noted before the onset of epididymitis.

From what has been stated, we may conclude that while tuberculosis of the prostate and vesicles may, and does, at times occur in children, it is certainly a far different situation than that which exists in the adult. In previous investigations of this question I found that in the adult these organs were definitely involved in seventy-six out of 101 cases, and I stated at that time that "the combined experience of all observers shows that prostatic and vesicular tuberculosis is most frequent during the time of the greatest activity of these organs, i. e., from about the twentieth to the fortieth years."

The comparative freedom from disease enjoyed by the infantile prostate may well account for the fact that I have nowhere seen it stated in the literature, nor has it been our own experience that bladder irritability or abnormal turinary constituents played a great part in the symptomatology of this disease in children. It also seems to be true that while in the adult renal tuberculosis sooner or later intervenes in a considerable number, constituting true "genito-urinary tuberculosis," this combination attacks the child with comparative rarity. In our eleven cases there was but one, a boy aged 8 , with renal tuberculosis, a complication which was discovered shortly after his first appearance at the hospital. In two cases the urine contained a few leukocytes and the slightest possible trace of albumin. Other than this there is no evidence in these cases of any damage to the urinary tract either before or after operation.

It is generally recognized that while the primary focus of tuberculosis in the urinary tract is in the kidney and while there are those who believe that in the genital tract this focus lies in the epididymis, it is equally certain that both foci are really secondary to a third. The statistics of all writers agree that this focus is generally in the lung or bronchial lymph nodes, and while it cannot always be demonstrated by the ordinary bedside methods of chest examination, the roentgen ray will reveal it in almost every instance. 
In the eleven cases here presented there was evidence of lung involvement in five, of tuberculous peritonitis in two, of chronic otitis media (probably tuberculous) in two, of bone tuberculosis in two, of cervical adenitis in one. In three cases no definite focus was established. It is obvious, therefore, that while disease of the lungs heads the list, it is equally clear that in a few there was evidence of active tuberculosis in more than one focus outside of the genital tract.

In view of these findings it is not surprising to observe that eight of the eleven patients were in bad condition when admitted to the hospital, loss of weight, anemia, rickets and malnutrition being predominant features. Such a large percentage of poor risks is in distinct contrast to the picture often presented by the adult suffering from a similar involvement of the genital tract. As often as not he is well nourished and superficially sound in appearance, the foci of disease being discovered sometimes only after the most painstaking search.

The surgical treatment of these cases consisted of orchidectomy in seven, and epididymectomy in two (of which one was bilateral). Of the remaining two one patient was unoperated and died of meningitis shortly after coming to the hospital, and the other was so occupied with the relief of his tuberculous peritonitis, and later of his empyema, that the treatment of his epididymis was left for another occasion.

A pathologic examination of the tissue removed was made in eight cases, all of which showed the characteristic lesions of tuberculosis. It is of interest to note that in six of these cases the presence of caseation indicated that the process was of long duration, much longer than would be supposed from the history obtained.

The outcome, more or less complete, is known in ten of these cases.

One child 2 years old, having left orchidectomy, was brought back the following year with a relapse on the opposite side. No further facts are known.

A boy, aged $61 / 2$ years, was seen a year after the removal of the right testicle and epididymis. His general and local condition were satisfactory as was that of the opposite testicle. The urine was negative.

Another boy, 12 years of age, returned a year after unilateral orchidectomy in excellent general condition.

A fourth child, 5 years of age, was seen two and a half years after bilateral epididymectomy. The note reads, "General condition excellent. Good appetite and has gained weight. Urine clean, negative. Has diurnal frequency. Scars not adherent to testes; no induration." This case is interesting as at the time of the operation the child had a bad interstitial keratitis and many râles in the lungs.

Another boy, 7 years old, was in very good general condition at the time of his unilaterel orchidectomy. He returned six years later 
when the following observation was made: "General condition good. Has gained in weight. No urinary symptoms. Right testis and epididymis normal. Prostate and vesicles normal. No fistula. Urine negative."

A sickly boy of 14 entered with tuberculous peritonitis, an old Pott's disease of the spine, and a unilateral epididymitis. After expectant treatment and being improved he was discharged. Nearly a year later he was readmitted for a cold abscess of the abdominal wall which was incised and drained. He was discharged in due time only to return three months later with empyema which was relieved by tapping. The condition of the epididymis had meantime grown somewhat worse but there was "nothing felt in the prostate." A few days later he was operated on for empyema, an operation from which he recovered fairly well. During the next three years he was a frequent visitor to the outpatient department, remaining in very fair condition. Since no note is made as to the condition of the genital tract it may be inferred that the disease was at least quiescent.

A boy, aged 8 , came in for relief of a scrotal abscess. Left epididymectomy was performed. A positive guinea-pig test of the bladder urine showed the presence of renal tuberculosis. Nearly three years later, the boy was readmitted and a left nephrectomy was performed. The opposite epididymis had meantime become involved.

During the next year or two he returned to the outpatient clinic and during this time he developed a tuberculous stricture of the bulbous urethra, which required frequent dilatation. It may be said also that he was meanwhile being given tuberculin and kept under good hygienic surroundings, yet in spite of these measures his decline was slow but steady. I have reason to believe he is now dead.

Three of these children are known to have died, a mortality of over 27 per cent., but a figure which does not by any means give the facts, as none have been followed a sufficient length of time. As I have already pointed out "my experience warrants the conclusion that until at least ten years have elapsed after operation, no patient can be said to be cured of genital tuberculosis." Further and more recent observations have only served to confirm the truth of this statement.

A child, aged 11/2 years, had unilateral orchidectomy. In addition to the genital tuberculosis, he had a chronic otitis media, cervical adenitis, and a tuberculous process of the ankle; altogether he was in poor shape. The wound had not healed at the time of his discharge, six weeks after operation. There is a note in the record to the effect that the patient died about four years later. The cause of death is not stated.

A necropsy was obtained in two cases. 


\section{REPORT OF CASES}

CASE 1.-History.-A boy, aged 6 years, with a family history of tuberculosis, is said to have had "meningitis" four years before entrance. For some time he had been in poor health, with cough, fever, abdominal pain and vomiting, loss of weight, and for two months he had had a swollen scrotum. There were no urinary symptoms.

Physical Examination.-Patient was a pale, sickly child, with definite tuberculosis of the left lung, and evidence of tuberculous peritonitis. The left scrotum was occupied by a large, hard, nodular mass. Orchidectomy was performed under ether. The pathologist stated that the "testis was studded with tubercles and the cord was found in the same condition." After a stormy convalescence, the patient developed tuberculous meningitis and died on the twenty-sixth day after operation.

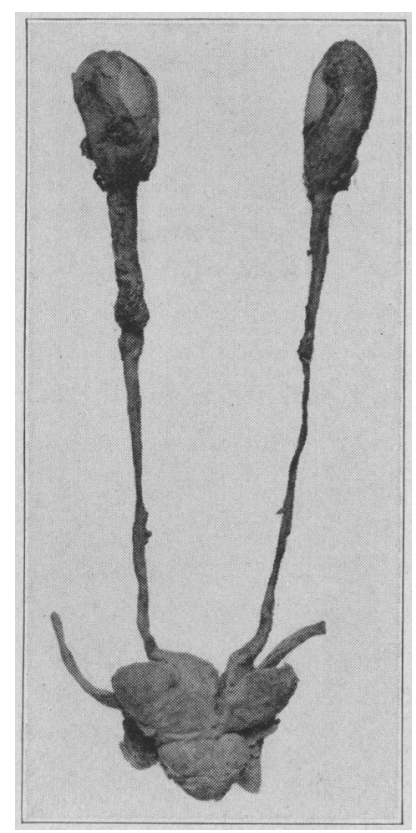

Fig. 1.-Entire genital tract of child 9 months of age. The middle portions of the vasa are of normal size. The seminal vesicles are greatly distended.

Necropsy.-Tuberculosis of the bronchial lymph glands, miliary tuberculosis of the lungs, liver, spleen and kidneys, tuberculous peritonitis, tuberculous meningitis, a solitary tubercle of the cerebellum, left otitis media, chronic pleuritis. The bladder, prostate, seminal vesicles and right testicle were "not remarkable."

CASE 2.-History.-A baby, aged 9 months, was seen December, 1920, in the Children's Medical Service at the Massachusetts General Hospital in consultation with Dr. F. B. Talbot. The family history was negative. He was well up to the seventh month when he developed a middle ear abscess followed by mastoiditis requiring radical operation. This process never entirely healed. Two months before coming to the hospital he developed a swelling of the left scrotum. At the time of entrance the child looked emaciated and sick. There was a chronic discharge from the ear and subsequent roentgen-ray examinations of the chest showed a glandular and peribronchial process, probably tuber- 
culous. The urine always contained albumin and a few leukocytes. Search for the tubercle bacillus was negative. There was moderate leukocytosis. Von Pirquet test was strongly positive. Both epididymes were swollen, especially the left, and firm and nodular. Scrotal skin adherent. Moderate tenderness.

Some days after entrance the child developed bronchitis and tuberculous meningitis to which it succumbed forty-six days after entrance.

Necropsy Report.-Tuberculous ulcers of the intestines, of the mesenteric, retroperitoneal, and bronchial lymph nodes, and miliary tuberculosis of the lungs, liver, spleen and kidneys. There was also meningeal tuberculosis.

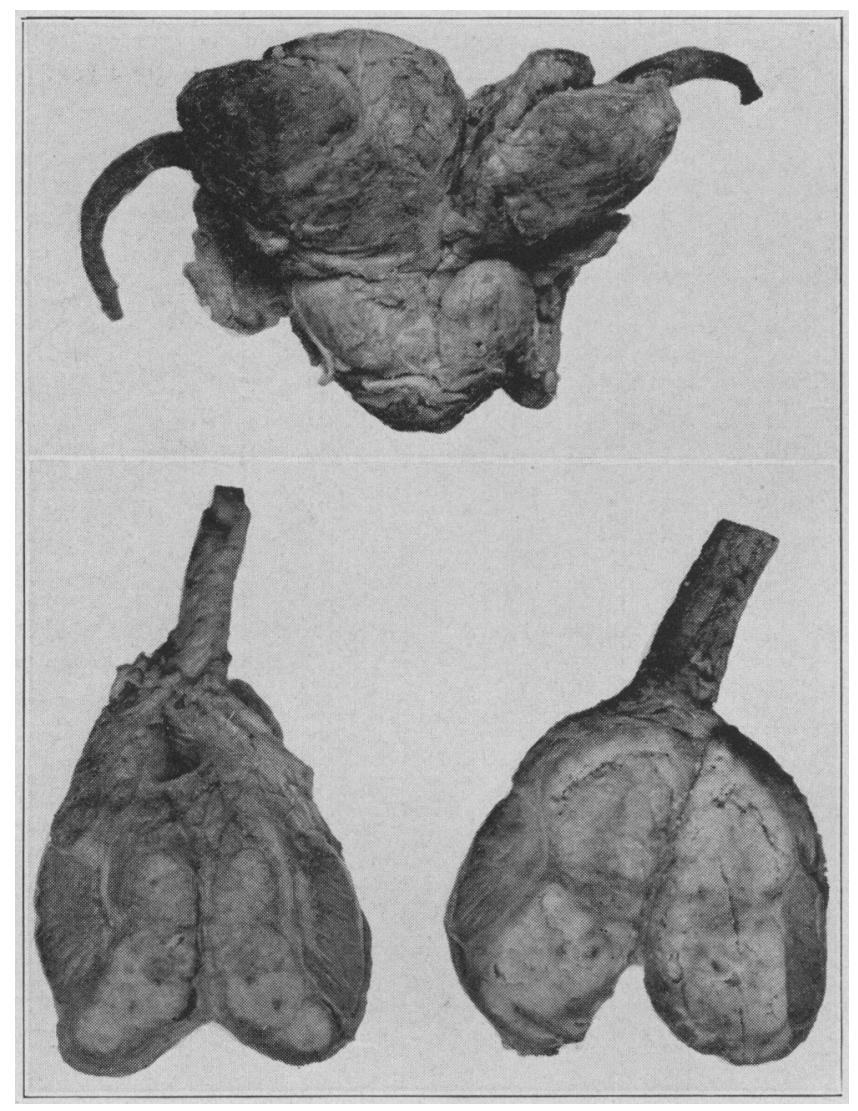

Fig. 2.-Enlarged view of prostate, vesicles and ampullae. Prostate is of normal size; vesicles and ampullae, especially the left, are enormously distended.

Fig. 3.-Testes and epididymes. Testes are entirely normal; epididymes are completely caseous and much enlarged.

The entire genital tract was dissected out by the pathologist, Dr. Oscar Richardson, to whom my thanks are due, as may be seen in Figures 1, 2 and 3.

The epididymes are much enlarged and caseous throughout. The testicles show no macroscopic changes. The vasa deferentia are thickened to the size of a goose quill at the outer third of their length, whereas in the middle third there is no gross abnormality. The ampullae are enormously distended. Both seminal vesicles, especially the left, are several times their normal size and on section are extensively caseous, there being but little normal tissue left. The 
prostate is of essentially normal size and appearance. On section it shows caseous areas within and immediately around the ejaculatory ducts throughout their entire course through the prostate. In one lateral lobe there is an illdefined, partly caseous area occupying about one-fourth of the lobe.

Microscopic study of these organs was made by Dr. James Homer Wright, to whom I express my sincere thanks.

Prostate: The opaque areas in the region of the ducts are typical ejaculatory tuberculosis, with much necrosis. The opaque area occupying about one quarter of the left lateral lobe shows small foci of necrosis in a cell-rich inflammatory tissue, in which are many epithelioid cells and some fibrous tissue. In the other lobe there are some small focal accumulations of epithelioid cells undergoing necrosis and constituting apparently recent tubercles. The microscopic appearance suggests a more recent process than in the ejaculatory ducts and in the epididymes (Figs. 4 and 5).

Epididymes: The enlarged caseous epididymes show appearances of tuberculosis of more long standing character. But little of the original tissue remains. As indicated by the gross appearance the tissue is largely necrotic, but actively growing tubercles are present at the margin of the necrotic mass in the fibrous inflammatory tissue. The testicles are normal.

Spermatic Cord: Sections from the median portion show appearance of tuberculosis.

Certain writers such as Vignard and Thévenot have attempted to divide genital tuberculosis into two groups, namely those in which the affection is essentially primary in the genital tract, and those in which this involvement is a secondary feature to visceral tuberculosis. I do not believe that this grouping is desirable or accurate as those placed in the second group are merely the more advanced stages of the first. The ultimate results of these cases would appear to confirm this view.

There seems also to be a considerable division of opinion as to treatment of this condition. While practically all are agreed that general hygienic measures, to which the use of tuberculin is added by some, are essential to the prospects of cure, the handling of the local disease is a bone of contention. Jullien, in 1889, and Rocher, in 1904, expressed strong objections to surgical intervention or only such as was absolutely necessary. On the other hand Felizet, in 1899, and Phocas, in 1898, advocated more radical measures involving. castration.

More recent writers, such as'Poissonnier, think it unwise to use anything more radical than the curette when the case is complicated by tuberculosis of other parts. Vignard and Thevenot think that those cases occurring in the first group in which the genital infection is a primary isolated manifestation, should be submitted only to the most conservative measures, whereas those in the second, with active lesions elsewhere, should have castration. They finally ask, however, if the difference between the primary and secondary forms is as great as one thinks.

As to the ultimate results of these cases in children Jullien says that he has lost none of twenty cases. Hutinel and Deschamps, on the 


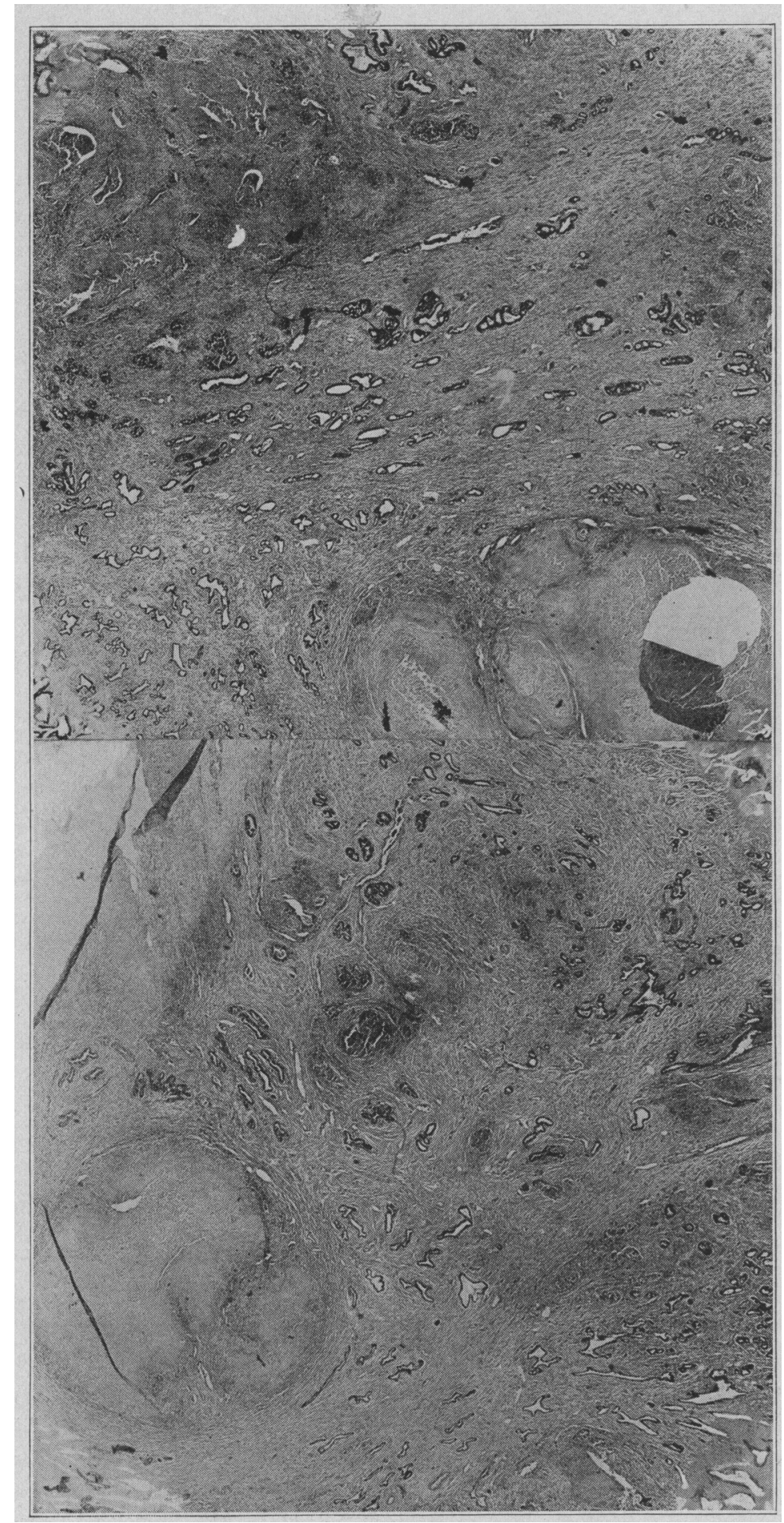

Fig. 4 (Cpper).-Left lobe of prostate, showing tuberculosis of the cjaculatory duct. The opaque area in upper portion shows foci of necrosis.

Fig. 5 (Lower).-Right lobe of prostate showing a few recent tubercles. 
contrary, have lost six out of nine cases. Vignard and Thévenot lost two out of their three patients, one dying six months, the other two months after the onset of the local infection. Most of the statistics on this point which I have seen are of little value, however, as there is no evidence that the patients have been followed for a sufficient length of time, an objection which applies to the cases I have here recorded. I have already expressed the opinion that until at least ten years have elapsed after operation, no patient can be said to be cured of genital tuberculosis.

Since the question of the primary focus and of the means by which the disease spreads from this focus to other organs is still open to question, I think that the views of two authoritative students of the problem, although at variance, are none the less interesting.

After many years of study of this question and basing his opinion on the results of 200 necropsies of those dying with genital or genitourinary tuberculosis Simmonds ${ }^{5}$ comes to the following conclusions:

1. The primary focus in the genital tract proceeds from the prostate in onehalf the cases, while in one-quarter of the cases it arises in the seminal vesicle or in the epididymis. This statement is based on forty necropsies in which he found the prostate alone involved in twenty, the seminal vesicles alone in ten, and the epididymis alone in ten.

2. From these centers the process can proceed in the direction of the testicle or away from it.

3. The process of healing seldom occurs in the prostate and seminal vesicles, while in the hilum of the testicle cicatrization is frequent.

4. The prognosis of male genital tuberculosis, particularly that of the seminal vesicle, is bad. The chief danger lies in the onset of tuberculous meningitis or of miliary tuberculosis. One third of the cases of genital tuberculosis in men die of this complication.

5. In the necropsies in twenty cases of men having previous castration for genital tuberculosis there was always found an involvement of the intraabdominal portion of the genital system. Healing was not observed in these cases.

6. Kraemer: Beitr. z. Klin. d. Tub. 33:259, 1914; 35:119, 1915; Deutsch. med. Wchnschr. 46:435, 1920 .

Simmonds himself acknowledges that there is a marked discrepancy between the clinical results and anatomic findings, but is unable to explain it. On the strength of his pathologic experience he advocates vesiculectomy, but feels that prostatectomy for tuberculosis is a dangerous procedure.

Kraemer, ${ }^{6}$ an equally authoritative and ardent student of the question, disagrees with Simmonds, basing his views on clinical as well as on anatomic findings. He believes that the spread of tuber-

5. Simmonds: Beitr. z. Klin. d. Tub. 33:35, 1914; 34:173, 1915.

6. Kraemer: Beitr. z. Klin. d. Tub. 33:259, 1914; 35:119, 1915; Deutsch. med. Wchnschr. 46:435, 1920 . 
culosis with the natural secretory current withis the male genital system holds true, and finds not only no evidence against this view in Simmonds' work, but even every evidence to support it. Regardless of what Simmonds found at necropsy, Kraemer believes that the undeniable fact that 80 per cent. of permanent cures of genital tuberculosis in the male reported by surgeons in general controverts any statement. If, he argues, the prostate is the primary focus, how can the removal of a secondary focus in the testicle produce this result. The difficulty lies in the fact that the patients seen by Simmonds had the misfortune to die and were among those the clinician failed to cure. Furthermore, as Simmonds himself acknowledges, the deaths are largely from miliary tuberculosis and meningitis, and these lesions might just as well be produced by the focus which gave rise to the genital infection, as by the genital infection itself.

Simmonds, in a subsequent article, refutes all of Kraemer's statements. He claims that one finds recent tuberculous changes in the epididymis along with far advanced changes in the prostate and vesicles. Also he thinks if the epididymis is the primary focus of genital tuberculosis, then isolated tuberculosis of the testicle should be met with more than isolated tuberculosis of the seminal vesicles and prostate, but this is not the case. Simmonds again acknowledges the great discrepancy between anatomical and clinical findings but again is unable to explain it. He concludes by remarking that whoever wishes to argue in favor of simple castration for genital tuberculosis should collect cases in which individuals so operated on will show no tuberculous changes after death.

To these arguments Kraemer replies with renewed vigor. He says it is useless to deny the report of many able surgeons that a clinical cure is effected in 80 per cent. of cases and to place against this indisputable evidence only 20 cases which were seen at necropsy. He thinks that the very fact that Simmonds himself cannot explain his findings is proof that his argument cannot hold water.

More recently Young, ${ }^{7}$ in a remarkably able and complete article, comes out with the assertion that genital tuberculosis arises not in the epididymis, nor in the prostate, but in the seminal vesicle.

Out of this welter of disagreement what are we to believe? In the first place, it is clear that those who have studied this question experimentally, clinically and anatomically are still uncertain as to the actual facts. In an experience of several years in the study of this problem I have found the most perplexing and conflicting evidence both clinical and anatomic. While I still believe that the great weight of evidence favors the origin of genital tuberculosis in the epididymis,

7. Young: Tr. Am. Assn. G.-U. Surg. 13:173, 1920 
I am willing to berieve that in certain instances the primary focus may lie in the prostate, possibly even in the seminal vesicle. It is impossible to take up and discuss seriatim and ad infinitum the argument of the various proponents of each point or origin, more especially those of Young. One can only make generalizations.

If genital tuberculosis arises in the prostate or even in the seminal vesical why is it that this disease is practically unknown clinically or postmortem? It is only reasonable to suppose that tuberculosis of either of these organs would produce symptoms for which relief would be sought and treatment given. Furthermore, prolonged observation of such cases would undoubtedly reveal the actual condition sooner or later. It is also true that in not a few instances necropsy has shown tuberculosis of almost every organ in the body, the prostate or seminal vesicles alone being spared.

If the prostate or seminal vesicle is the starting point of epididymal tuberculosis why is it that the removal of the epididymis has such a salutary effect on the other organs of the genital tract? I have followed a good many cases for a good many years both before and after operation. While there have been occasional exceptions, as is to be expected, the prostate and vesicles which were nodular, indurated and enlarged before epididymectomy have eventually returned to an essentially normal condition. It is probably true that while the microscope would still show evidence of tuberculosis in some, it is equally true that fibrous changes eventually occur and a clinical and permanent cure is established. This statement is not a case of the wish being father to the thought, but is an actual statement of fact.

In this connection one cannot avoid touching on the subject of treatment. The results just mentioned have occurred after epididymectomy or orchidectomy, yet there are those, of whom Young is the most recent and ardent, who urge the desirability of and the necessity for the removal of the entire genital tract. While this operation certainly gives free scope to the skill, patience and ingenuity of the surgeon, it apparently does not seem to afford an equal amount of satisfaction to the patient and after all, surgery should have this as one of its objects. If the prostate will quiet down spontaneously by a simple procedure why remove the prostate? Also is it not against all surgical principles for a primary focus to subside after the removal of a secondary focus. Why not remove the entire ureter and the bladder as well as the kidney in treating renal tuberculosis? The procedure would be quite as logical. Or why not cure cancer of the breast by dissecting out the glands in the axilla?

If the primary focus lies in the prostate or seminal vesicle why are these organs less frequently attacked in children than in adults? 
Yet this seems to be the fact judging from my own experience and from the reports in the literature.

The radical operation of removing the entire genital tract is thought to reduce the immediate as well as the ultimate mortality. Does it do so? In the past ten years more than 100 cases of genital tuberculosis have been admitted to the genito-urinary service of the Massachusetts General Hospital. All but a few patients have had either epididymectomy or orchidectomy performed. There has been one death in the hospital in this time, and this patient was not operated on. It would not appear, therefore, that conservative surgery involved a high death rate. It is true that the ultimate deaths from tuberculosis are high and that at least ten years must elapse before the danger of dying from tuberculosis is past. Many of the deaths are from lung or general miliary tuberculosis. The radical surgeons claim that this high mortality is due to the tuberculous genitals which the conservative surgeons leave behind, yet they have presented no evidence that this mortality did not arise quite as much from the original process in the lung or other organs which induced the genital tuberculosis, as from the genital tuberculosis itself. Furthermore, few, if any, of their cases have been followed a sufficient length of time to show what the outcome will be. Certainly the results reported by Whiteside ${ }^{8}$ are far from encouraging.

The foregoing discussion may not be entirely irrelevant to the subject of genital tuberculosis in children. It has been shown that these little patients are somewhat differently affected than are the adults, but after all their hematogenous and lymphatic channels are the same, they are equally susceptible if not more so than adults to infection, and yet the prostate and seminal vesicles still in a state of inactivity do not seem to be as frequently affected by tubercu'osis as do the adult organs.

8. Whiteside: Northwest Med. 18:83-85, 1919. 